\title{
水相中可见光催化腈合成苯并咪唑衍生物
}

\author{
林 媚*,a 吴 凡 ${ }^{a}$ 刘天惠 ${ }^{a}$ 陈志涛 ${ }^{a}$ 许秀枝 ${ }^{a}$ 柯 方*,a,b \\ $\left({ }^{a}\right.$ 福建医科大学药学院 福州 350004) \\ ${ }^{b}$ 福建省天然药物药理学重点实验室 福州 350004)
}

\begin{abstract}
摘要 报道了在水相中可见光下 1,2 -苯二胺与苯腈的环化反应, 合成了一系列苯并咪夾类化合物. 反应用便宜、易处 理、对环境无污染的曙红 Y 作为光催化剂. 室温下在水相中反应，获得的产物有较好收率，最高产率可达到 $91 \%$. 提供 了一种合成苯并咪唑类化合物的简便经济的方法.
\end{abstract}

关键词 可见光; 曙红 Y; 苯并咪唑; 催化剂

\section{Visible-Light Promoted Preparation of Benzimidazoles by Eosin $Y$ Catalyzed Reaction of Benzonitrile Derivatives in Water}

\author{
Lin, Mei ${ }^{*, a} \quad \mathrm{Wu}$, Fan $^{a} \quad$ Liu, Tianhui $^{a} \quad$ Chen, Zhitao $^{a} \quad \mathrm{Xu}$ Xiuzhi $^{a} \quad$ Ke, Fang ${ }^{*, a b}$ \\ ( ${ }^{a}$ Department of Pharmacy, Fujian Medical University, Fuzhou 350004) \\ $\left({ }^{b}\right.$ Fujian Provincial Key Laboratory of Natural Medicine Pharmacolog, Fuzhou 350004)
}

\begin{abstract}
A novel visible-light-introduced reaction for the construction of benzimidazole derivatives via radical cyclization of $o$-phenylenediamines with benzonitrile derivatives in water has been developed. The reaction has been achieved in high yield under mild conditions by using Eosin $\mathrm{Y}$ as photocatalyst, which is cheap, easy to handle and environmentally friendly. A variety of benzimidazoles were obtained in up to $91 \%$ yields. It might provide a promising protocol for the synthesis of benzimidazole derivatives.
\end{abstract}

Keywords visible-light photoredox catalysis; eosin Y; benzimidazole; catalyst

苯并咪唑类化合物是许多碱性天然产物和众多具 有生物活性药物的分子骨架，此外苯并咪唑类 C-2 位取 代基的类型、空间构型不同, 都使此类化合物具有不同 的药用活性 ${ }^{[1]}$. 例如宠物心脏病肥大、宠物心力衰竭药 Pimobendan 和驱虫药 Vermox 等(图 1). 因此, 苯并咪坐 结构的合成便成为无数有机和药物化学家非常感兴趣 的领域之一.

目前合成此类化合物的传统途径主要有以下几种: 一种是在强酸作用下, 如盐酸等, 由 1,2 -苯二胺及其衍 生物与羧酸类化合物反应制得; 该法通常需要较高的反 应温度或较长的反应时间, 效果不理想, 其次对生产设 备要求较高 ${ }^{[2]}$. 另一种则是通过 1,2-苯二胺衍生物与醛 类化合物在氧化剂的作用下环合而得; 该反应过程比较 简便, 但副产物较多, 分离困难 $(S c h e m e 1 \mathrm{a})^{[3]}$. 近年来,<smiles>COc1ccc(-c2nc3cc(C4=NNC(=O)CC4C)ccc3[nH]2)cc1</smiles>

图 1 一些具有苯并咪唑结构的活性分子

Figure 1 Some active molecules with benzimidazole moieties 在传统的合成路线基础上，相继出现了一系列以路易斯 酸和过渡金属络合物等为催化剂的新合成路线, 取得了 较好的效果 ${ }^{[4]}$. 此外采用其他原料合成苯并咪唑的方法

* Corresponding authors. E-mail: linmei@fjmu.edu.cn; kefang@163.com Received April 11, 2020; revised May 9, 2020; published online May 25, 2020.

Project supported by the Natural Science Foundation of Fujian Province (Nos. 2016Y9052, 2016Y9053, 2017J01820). 福建省自然科学基金(Nos. 2016Y9052, 2016Y9053, 2017J01820)资助项目. 
也有报道 ${ }^{[5]}, 2013$ 年, 周向葛课题组 ${ }^{[6]}$ 以商业易得的 $N, N^{\prime}$-二甲基乙二胺为配体, $\mathrm{Cu}_{2} \mathrm{O}$ 为催化剂, 发现了以 2卤代芳胺与脒盐为原料合成苯并咪唑衍生物的方法, 大 量苯并咪唑衍生物被高效合成出来. 2013 年, Xiang 课题 组 ${ }^{[7]}$ 发现 $N$-芳基酰胺可以由卤代芳烃和腈发生取代反 应制备(Scheme 1b). 受此启发, 目前各种 $N$-芳基酰胺苯 并咪唑衍生物可以根据这种方法.

(a)

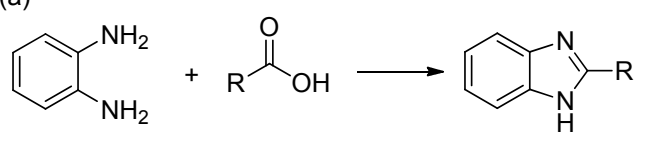

(b)

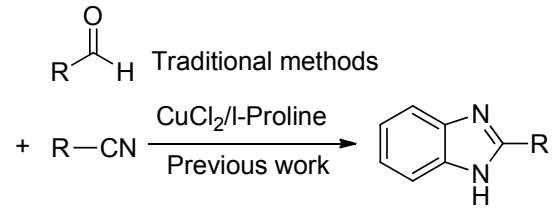

(c)

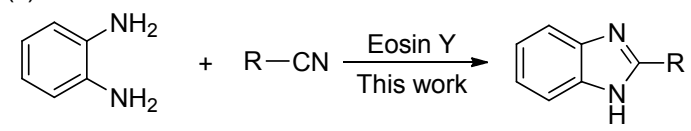

图式 1 苯并咪唑的合成方法

Scheme 1 Strategies for the synthesis of benzimidazoles

腈类化合物结构比较简单, 却拥有十分丰富的化学 性质. 首先氰基是一个很好的离去基团, 可以被一些亲 核试剂取代; 其次在不同的反应条件下，氰基作为含氮 杂环的氮源, 能选择地参加反应 ${ }^{[8]}$, 这使得腈类化合物 表现出异乎寻常的化学反应性质, 并被广泛深入的研 究, 但是利用腈为原料制备苯并咪唑的研究却较少. 2014 年, $\mathrm{Lu}$ 等 ${ }^{[9]}$ 发现苯并咪唑可以通过 2-碘苯胺和苯腈 为原料, 在铜催化剂下可有效进行反应, 但是产率不 高、反应时间过长等缺点限制了其发展.

1972 年, Fujishima 和 Honda ${ }^{[10]}$ 在 $\mathrm{n}$ 型半导体 $\mathrm{TiO}_{2}$ 电极上实现了水的光电催化分解, 自此光催化正式进入 人类的视野. 光催化反应是在光和催化剂的同时作用下 所进行的化学反应, 是光反应和催化反应的融合. 与传 统的金属催化、有机小分子催化以及酶催化相比较, 光 催化剂具有用量少、反应条件温和、底物适用性好以及 实验操作简单等优点, 不仅符合当今绿色化学主题, 而 且在理论和实际应用上具有非常重要的科学意义 ${ }^{[11]}$. 光 催化已经在有机化学中得到广泛关注. 水具有无毒、环 境友好、廉价、易得等绿色反应介质的所有特性. 用水 来代替有机溶剂, 符合当今绿色化学发展的主题. 目前 为止, 绝大多数光催化反应都是在有机相中进行, 在纯 水相中进行可见光介导的有机反应报道鲜少 ${ }^{[12]}$. 2017 年, Kumar 小组 ${ }^{[13]}$ 以 Langlois 试剂和 9,10-二酮菲蒽作为 光氧化还原催化剂, 水作为氧源, 实现了光诱导氧化、
无金属串联自由基环化、三氟甲基化和 1,6-烯炔的脱氢 氧化反应. 该方案可以得到多种含 $\mathrm{CF}_{3}$ 取代基的杂环化 合物..

基于本课题组前期对环境友好型催化体系的研 究 ${ }^{[14]}$, 本文报道一种用廉价易得、易处理的曙红 $Y$ 作为 光催化剂, 1,2-苯二胺与苯腈为原料, 在水相中高效合 成一系列苯并咪唑衍生物的反应，为苯并咪唑类化合物 的合成提供了一种简便经济的方法(Scheme 1c).

\section{1 结果与讨论}

\section{1 反应条件的优化}

以 1,2-苯二胺、苯甲腈为原料, $\mathrm{CuI}$ 为催化剂, 1,10 菲啰啉 $(\mathbf{L 1}, 1,10-P h e n)$ 为配体, 氢氧化钾 $(\mathrm{KOH})$ 为碱, 在 室温下用 $25 \mathrm{~W}$ 白色节能灯照射, 在纯水溶剂中考察了 一系列光催化剂对反应的影响. 结果如表 1 所示: 当使 用 $\operatorname{Ir}(\mathrm{bpy})_{3}$ 为光敏剂时，在 $25 \mathrm{~W}$ 的白色节能灯照射下， 反应可以获得 $80 \%$ 的收率; 当使用有机光敏剂曙红 (Eosin $\mathrm{Y}$ ) 时, 获得 $83 \%$ 的收率; 但使用有机光催化剂 2,4,6-三苯基吡喃四氟硼酸盐(TPT)时，却达不到预期效 果(表 1, Entries 1 3). 通过条件控制实验证明(表 1, Entry 4), 可见光光照以及光催化剂是保证该反应顺利 进行不可或缺的因素, 确定了该反应是可见光诱导的环 化反应. 随后, 以 Eosin $\mathrm{Y}$ 作为光催化剂, 考察了不同的 铜源, 如 $\mathrm{CuCl}_{2} 、 \mathrm{CuSO}_{4}$ 和 $\mathrm{CuCl}$ 等. $\mathrm{CuCl}_{2}$ 表现出最好的 催化效果(表 1, Entries 6,7). 有趣的是, 如果不使用铜 盐, 只能收获痕量的目标产物, 我们认为铜盐在这个催 化体系中能很好地降低催化门槛(表 1, Entry 8). 进一步 探索优化配体发现, 通过研究不同配体和 $\mathrm{CuCl}_{2}$ 的相互 作用, $\mathbf{L 1}$ 配体在光催化合成苯并咪唑的反应中于 $\mathrm{CuCl}_{2}$ 能更好地促进反应的进行(表 1, Entries 9 11). 该催化 体系在缺少配体的情况下反应效果很差(表 1, Entry 12). 对碱进行篎选发现, $\mathrm{Cs}_{2} \mathrm{CO}_{3}$ 作碱时，反应能以高达 $90 \%$ 的分离收率得到目标化合物(Entries 13 15). 此外, 研 究发现反应催化过程中相转移催化剂(PTC) 是必要的, 而且四丁基溴化铵(TBAB)的收率明显优于四丁基碘化 铵(TBAI) (Entries 16, 17). 因此, 从实验结果中得出该 反应体系的最佳催化条件为: 1,2 -苯二胺 $(0.5 \mathrm{mmol})$, 苯 腈 $(0.6 \mathrm{mmol})$, Eosin Y (5 mol\%), $\mathrm{CuCl}_{2}(0.05 \mathrm{mmol})$, 配 体 $\mathbf{L 1}(0.05 \mathrm{mmol}), \mathrm{Cs}_{2} \mathrm{CO}_{3}(1 \mathrm{mmol}), \mathrm{TBAB}(0.05 \mathrm{mmol})$, $2 \mathrm{~mL}$ 水作为溶剂, $25 \mathrm{~W}$ 可见光, 反应 $7 \mathrm{~h}$.

\section{2 反应条件的优化}

为了探索该催化体系的适用范围, 选择了一系列取 代的 1,2-苯二胺与各种苯腈在纯水相中 $25 \mathrm{~W}$ 白光进行 光照, 对底物的普适性进行考察, 结果见表 2 . 结果发 
表 1 反应条件的优化 ${ }^{a}$

Table 1 Optimization of the reaction conditions

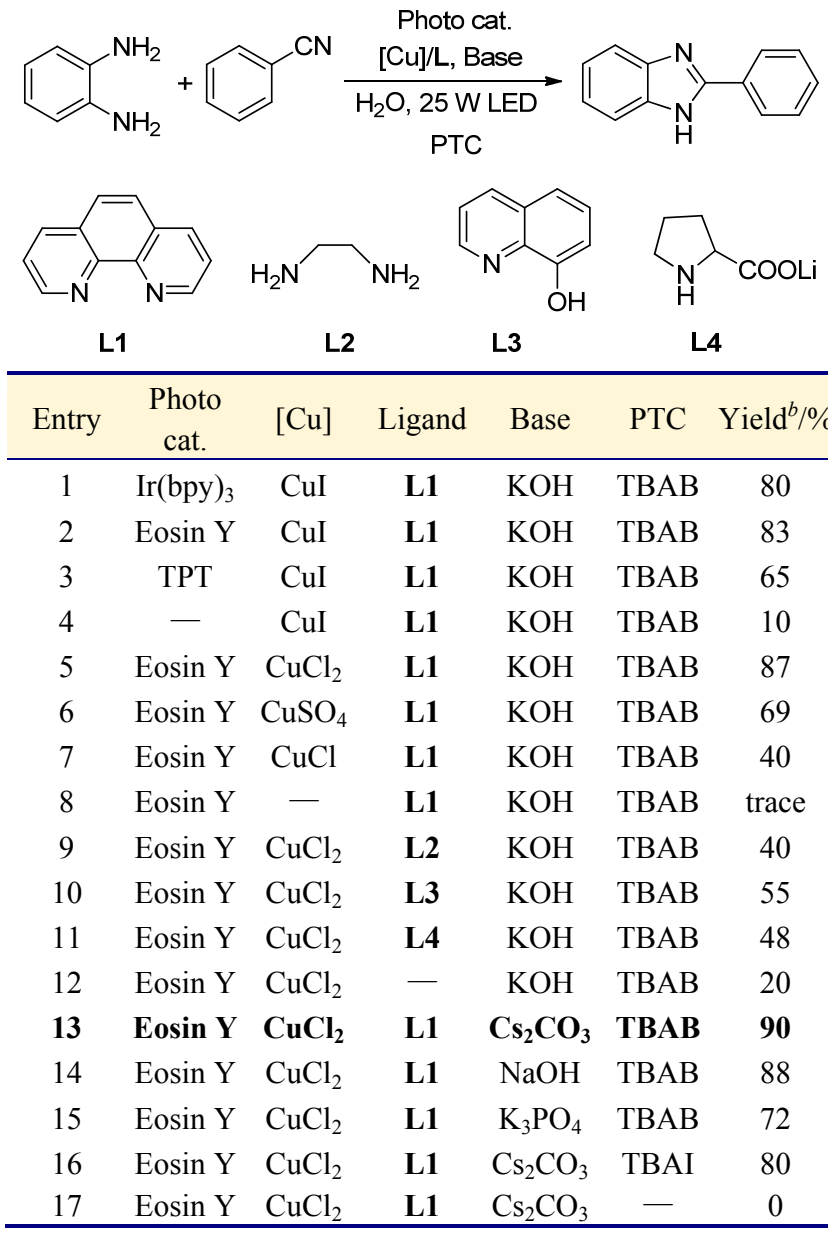

${ }^{a}$ Reaction conditions: 1,2-diaminobenzene $(0.5 \mathrm{mmol})$, benzonitrile $(0.6$ $\mathrm{mmol})$, photo catalyst $(5 \mathrm{~mol} \%)$, Cu source $(0.05 \mathrm{~mol})$, ligand $(0.05 \mathrm{~mol})$, base (1 mmol), PTC (0.05 mmol), and $\mathrm{H}_{2} \mathrm{O}(2 \mathrm{~mL}), 25 \mathrm{~W} \mathrm{LED}, 7 \mathrm{~h} ;{ }^{b}$ Isolated yield.

现该催化体系对于各种 1,2-苯二胺和苯腈均具有较 高的催化活性, 大部分底物均能以较好的收率专一地生 成相应目标产物, 无论是供电子基团还是吸电子基团, 如甲基、甲氧基、氟、氯、澳、硝基和羟基等, 都能获 得较好收率的苯并咪唑化合物. 首先将各种不同取代基 的苯腈与 1,2-苯二胺进行反应, 发现含供电子取代基的 苯腈的产率略高于吸电子取代基的苯腈, 如对位是甲氧 基取代苯腈，与 1,2-苯二胺反应产率可达 $91 \%$, 而对硝 基取代苯腈反应，仅得到 72\%产率(3b, 3o). 其次含供电 子基团的 1,2-苯二胺的活性高于含吸电子基团的 1,2-苯 二胺, 因为供电子性对胺基正离子和自由基电子具有稳 定作用. 如 4-硝基-1,2-苯二胺与苯腈反应的产率为 71\% (3I)，4-甲基-1,2-苯二胺与苯腈反应的产率为 $81 \%$ (3m). 此外, 该体系对脂肪族腈类化合物同样适用, 产率略低 于芳香族腈类 $(\mathbf{3} \mathbf{j})$. 最后, 该体系对含杂环的腈类同样 适用, 如采用 2-氧基吡啶和 1,2-苯二胺为原料, 得到产 率为 75\%的 2-(吡啶-2-基)-1 $H$-苯并[d]咪唑(3n).
表 2 苯并咪唑衍生物的合成 ${ }^{a}$

Table 2 Synthesis of benzimidazoles

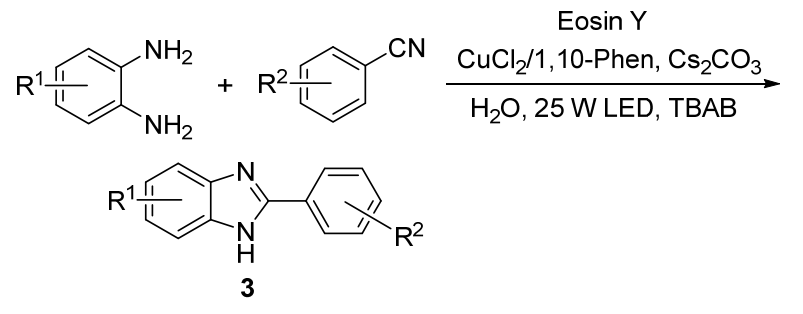

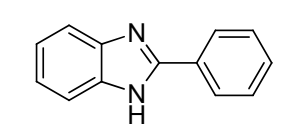

3a $90 \%^{b}$<smiles>COc1ccc(-c2nc3ccccc3[nH]2)cc1</smiles>

3c $91 \%$<smiles>Brc1ccc(-c2nc3ccccc3[nH]2)cc1</smiles>

3e $78 \%$

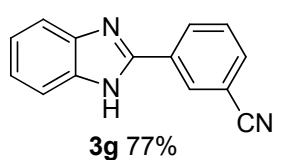

3g $77 \%$<smiles>Oc1ccccc1-c1nc2ccccc2[nH]1</smiles>

3i $78 \%$<smiles>Clc1cc2nc(-c3ccccc3)[nH]c2cc1Cl</smiles>

3k $73 \%$<smiles>Cc1ccc2nc(-c3ccccc3)[nH]c2c1</smiles><smiles>O=[N+]([O-])c1ccc(-c2nc3ccccc3[nH]2)cc1</smiles><smiles>Cc1ccc(-c2nc3ccccc3[nH]2)cc1</smiles>

3b $91 \%$<smiles>Clc1ccc(-c2nc3ccccc3[nH]2)cc1</smiles><smiles>COc1cc(-c2nc3ccccc3[nH]2)ccc1O</smiles>

3f $91 \%$<smiles>Fc1ccccc1-c1nc2ccccc2[nH]1</smiles>

3h $71 \%$<smiles>CC(C)c1nc2ccccc2[nH]1</smiles>

3j $72 \%$<smiles>O=[N+]([O-])c1ccc2nc(-c3ccccc3)[nH]c2c1</smiles>

$3171 \%$<smiles>c1ccc(-c2nc3ccccc3[nH]2)nc1</smiles>

3n $75 \%$

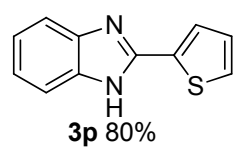

${ }^{a}$ Reaction conditions: $o$-phenylenediamines $(0.5 \mathrm{mmol})$, benzonitriles $(0.6$ $\mathrm{mmol})$, Eosin $\mathrm{Y}(5 \mathrm{~mol} \%), \mathrm{CuCl}_{2}(0.05 \mathrm{~mol}), \mathbf{L}_{1}(0.05 \mathrm{~mol}), \mathrm{Cs}_{2} \mathrm{CO}_{3}(1 \mathrm{mmol})$, TBAB $(0.05 \mathrm{mmol})$, and $\mathrm{H}_{2} \mathrm{O}(2 \mathrm{~mL}), 25 \mathrm{~W} \mathrm{LED}, 7 \mathrm{~h} ;{ }^{b}$ Isolated yield.

\section{3 克级反应}

依据最优反应条件，对底物进行克级放大实验. 以 1,2 -苯二胺 $(5 \mathrm{mmol})$, 苯甲腈 $(6 \mathrm{mmol})$ 为原料, Eosin $\mathrm{Y}(5$ $\mathrm{mol} \%), \mathrm{CuCl}_{2}(0.5 \mathrm{mmol})$, 配体 $\mathbf{L 1}(0.5 \mathrm{mmol})$ 为催化剂, $10 \mathrm{mmol} \mathrm{Cs}_{2} \mathrm{CO}_{3}$ 作为碱, $0.5 \mathrm{mmol} \mathrm{TBAB}$ 作为添加剂, $20 \mathrm{~mL}$ 水作为溶剂, $25 \mathrm{~W}$ LED 白光照射下反应 $10 \mathrm{~h}$, 能 顺利生成 2-苯基- $1 H$-苯并 $[d]$ 咪唑, 产率为 $66 \%$. 


\section{4 机理探索}

为了探索反应机理, 进行了自由基捕获实验 (Scheme 2). 依据最优反应条件, 以 1,2-苯二胺 $(0.5$ $\mathrm{mmol})$, 苯甲腈 $(0.6 \mathrm{mmol})$ 为原料, Eosin $\mathrm{Y}(5 \mathrm{~mol} \%)$ 、 $\mathrm{CuCl}_{2}(0.05 \mathrm{mmol})$, 配体 $\mathbf{L 1}(0.05 \mathrm{mmol})$ 为催化剂, 1 $\mathrm{mmol} \mathrm{Cs}_{2} \mathrm{CO}_{3}$ 作为碱, $0.05 \mathrm{mmol} \mathrm{TBAB}$ 作为添加剂, 2 $\mathrm{mL}$ 水作为溶剂, 加入 2.0 equiv. 2,2,6,6-四甲基哌啶-1氧(TEMPO), 这个反应被终止. 遗憾的是没有得到捕获 产物, 因此认为捕获产物应该为铜盐复合物. 为了验证, 在标准反应条件下分别加入 2.0 equiv. 2,6-二叔丁基对 甲酚(BHT)和 1,1-二苯基乙烯进行反应，也都没有得到 捕获产物. 所以进一步印证捕获产物应该为铜盐复合 物.

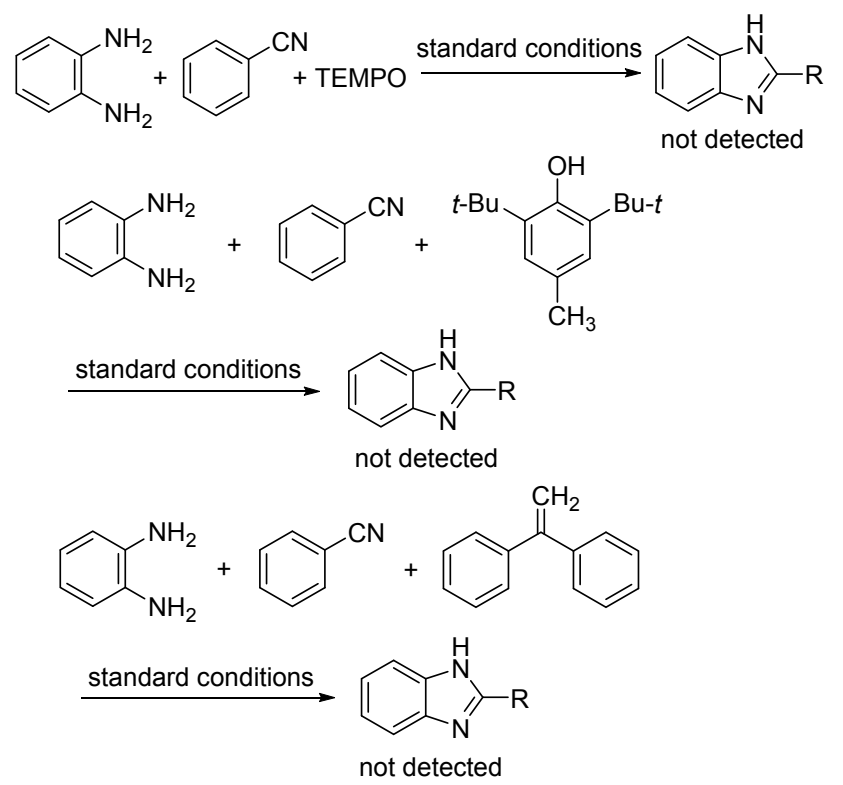

图式 2 自由基捕获实验

Scheme 2 Radical trapping experiments

\section{5 反应机理}

根据实验结果和文献报道 ${ }^{[15-17]}$, 提出本实验过程可 能发生的反应机理(Scheme 3). 铜盐在碱性条件下和配 体形成铜盐配体复合物, 然后作为路易斯酸和苯腈形成 中间体苯腈-铜(II)盐配体复合物. 同时, 曙红 Y 吸收光 子变为激发态的曙红 $\mathrm{Y}$, 然后处于激发态的曙红 $\mathrm{Y}$ 给出 一个电子给溴离子, 生成了溴自由基. 随后苯腈-铜(II) 盐配体复合物受到溴自由基的进攻, 生成中间体溴苯 氨一铜(II)盐配体复合物 $\mathbf{A}$. 紧接着, 中间体 $\mathbf{A}$ 与邻苯二 胺反应生成瞬时中间体 $\mathbf{B}$. 中间体 $\mathbf{B}$ 分子内电子发生转 移进行了分子内消除耦合产生中间体 C. 最后在反应条 件下脱去一分子氨基铜盐配体复合物, 生成苯并咪唑. 脱去的氨基铜盐配体复合物进入光循环, 被还原成一分 子的氨和铜(II)盐配体复合物.
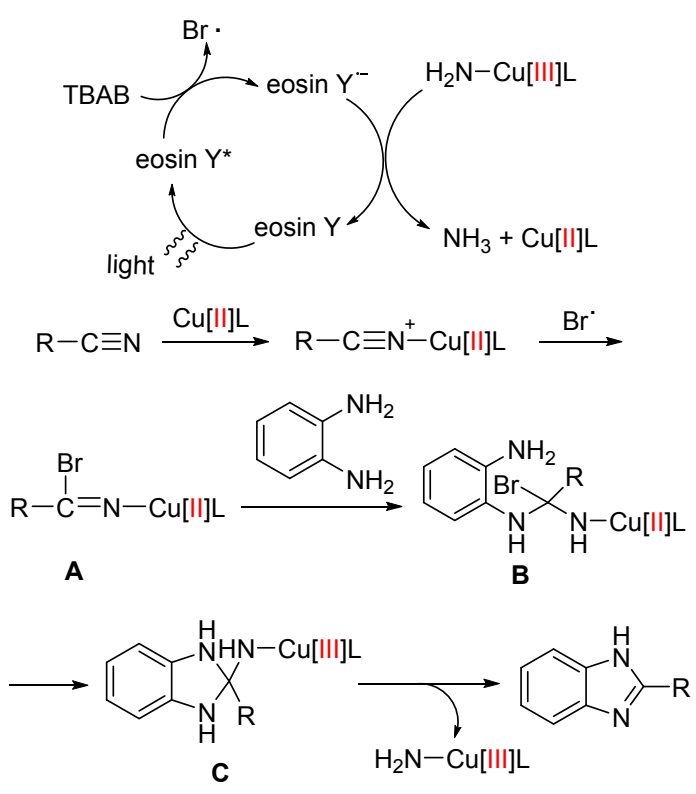

图式 3 可能发生的反应机理

Scheme 3 Proposed mechanism for this transformation

\section{2 结论}

通过用曙红 $\mathrm{Y}$ 作光催化剂, 实现了以 $\mathrm{CuCl}_{2}$ 作金属 催化剂和 1,10-菲啰啉作配体, 以 1,2-苯二胺与苯腈取代 物为原料, 水相中自由基环化反应合成苯并咪唑衍生物 的方法. 反应用曙红 Y 作为光催化剂，价格便宜、易处 理、对环境无污染, 利用纯水作为溶剂, 较大地降低了 反应成本. 该反应为一系列通过苯腈合成苯并咪唑衍生 物的合成提供了一种更简便经济的方法，更多关于可见 光催化的自由基环化反应的研究正在我们实验室进行 中.

\section{3 实验部分}

\section{1 仪器与试剂}

光反应器: Perfectlight PCX50C Discover; 质谱仪: Agilent $6890 \mathrm{~N}$ 进行气相色谱/质谱(GC/MS)测试 [5973N 质量检测器 $(E I)$ 和 HP5-MS $30 \mathrm{~m} \times 0.25 \mathrm{~mm}$ 毛细管非极 性柱]; 核磁共振仪: Bruker AM 400, 以 TMS 为内标, 氛 代 DMSO 等为溶剂; X-4 型数字熔点仪. 柱色谱用硅胶 (200 300 目)进行，乙酸乙酯和石油醚为洗脱剂. 硅胶 GF254 板进行薄层色谱. 所用的药品和试剂均为市售分 析纯或化学纯, 除特别说明外, 未经进一步处理.

\section{2 实验方法}

在 $10 \mathrm{~mL}$ 的圆底烧瓶中加入 1,2 -苯二胺 $(0.5 \mathrm{mmol})$, 苯甲腈 $(0.6 \mathrm{mmol})$, 曙红 $\mathrm{Y}$ (0.05 equiv.), $\mathrm{CuCl}_{2}(0.05$ $\mathrm{mmol}) 、$ 配体 1,10-Phen $(0.05 \mathrm{mmol}) 、 \mathrm{Cs}_{2} \mathrm{CO}_{3}(1 \mathrm{mmol})$ 、 $\operatorname{TBAB}(0.05 \mathrm{mmol})$ 和溶剂 $\mathrm{H}_{2} \mathrm{O}(2 \mathrm{~mL})$. 反应液在一个 25 
$\mathrm{W}$ 的白光 LED 灯下 (距离反应瓶 $5 \mathrm{~cm}$ ) 在光反应器内室 温摚拌 $7 \mathrm{~h}$. 反应用薄层色谱(TLC)检测, 反应完全后, 减压蒸馏, 粗产品通过柱层析分离纯化 $[V$ (乙酸乙酯) : $V($ 石油醚 $)=3: 1$ ] 得到目标产物, 计算产率. 目标产物 通过 ${ }^{1} \mathrm{H}$ NMR, ${ }^{13} \mathrm{C}$ NMR 和质谱进行结构表征.

2-苯基-1 $H$-苯并 $[d]$ 咪唑(3a)：白色固体, m.p. 294 $296{ }^{\circ} \mathrm{C}$ (Lit. ${ }^{[16 a]}$ : 293 $295{ }^{\circ} \mathrm{C}$ ); ${ }^{1} \mathrm{H}$ NMR $(400 \mathrm{MHz}$, acetonitrile- $\left.d_{3}\right) \delta: 10.92(\mathrm{~s}, 1 \mathrm{H}), 8.10(\mathrm{~d}, J=4.0 \mathrm{~Hz}, 2 \mathrm{H})$, $7.56(\mathrm{dd}, J=21.2,13.6 \mathrm{~Hz}, 5 \mathrm{H}), 7.23 \sim 7.26(\mathrm{~m}, 2 \mathrm{H}) ;{ }^{13} \mathrm{C}$ NMR (100 MHz, DMSO- $\left.d_{6}\right) \delta: 151.2,143.8,135.0,130.1$, $129.8,128.9,126.4,122.5,121.6,118.8,111.3$; ESI-MS $m / z: 195.1[\mathrm{M}+\mathrm{H}]^{+}$.

2-(对甲苯基)- $1 H$-苯并 $[d]$ 咪唑(3b): 白色固体, m.p. $278 \sim 279{ }^{\circ} \mathrm{C}$ (Lit. ${ }^{[16 a]}: 276 \sim 278{ }^{\circ} \mathrm{C}$ ); ${ }^{1} \mathrm{H}$ NMR $(400$ $\mathrm{MHz}$, acetonitrile- $\left.d_{3}\right) \delta: 12.23(\mathrm{~s}, 1 \mathrm{H}), 7.98(\mathrm{~d}, J=8.0 \mathrm{~Hz}$, 2H), 7.59 (s, 2H), 7.36 (d, $J=8.0 \mathrm{~Hz}, 2 \mathrm{H}), 7.23$ (dd, $J=$ 5.8, $3.0 \mathrm{~Hz}, 2 \mathrm{H}), 2.41(\mathrm{~s}, 3 \mathrm{H}) ;{ }^{13} \mathrm{C}$ NMR $(100 \mathrm{MHz}$, DMSO- $\left.d_{6}\right) \delta: 144.7,142.6,135.7,133.8,130.2,129.7$, $129.0,126.5,122.5,120.1,118.2,112.6$; ESI-MS $\mathrm{m} / \mathrm{z}$ : $209.1[\mathrm{M}+\mathrm{H}]^{+}$.

2-(对甲氧基苯基)- $1 H$-苯并 $[d]$ 咪唑(3c)：白色固 体, m.p. $222 \sim 223{ }^{\circ} \mathrm{C}$ (Lit. ${ }^{[16 a]}$ : $221 \sim 223{ }^{\circ} \mathrm{C}$ ); ${ }^{1} \mathrm{H}$ NMR $\left(400 \mathrm{MHz}\right.$, acetonitrile- $\left.d_{3}\right) \delta: 11.06(\mathrm{~s}, 1 \mathrm{H}), 8.03(\mathrm{~d}, J=8.0$ $\mathrm{Hz}, 2 \mathrm{H}), 7.61 \sim 7.52(\mathrm{~m}, 2 \mathrm{H}), 7.21(\mathrm{dd}, J=5.8,3.0 \mathrm{~Hz}$, 2H), 7.07 (d, $J=8.0 \mathrm{~Hz}, 2 \mathrm{H}), 3.86$ (s, 3H); ${ }^{13} \mathrm{C}$ NMR $(100$ MHz, DMSO- $\left.d_{6}\right) \delta$ : $160.6,151.3,143.9,134.9,128.0$, $122.7,122.0,121.4,118.5,114.3,111.0,55.3$; ESI-MS $\mathrm{m} / \mathrm{z}: 225.1[\mathrm{M}+\mathrm{H}]^{+}$.

2-(4-氯苯基)- $1 H$-苯并 $[d]$ 咪唑(3d): 白色固体, m.p. $304 \sim 305{ }^{\circ} \mathrm{C}$ (Lit. $\left.{ }^{[16 \mathrm{f}]}: 302 \sim 303{ }^{\circ} \mathrm{C}\right) ;{ }^{1} \mathrm{H}$ NMR $(400$ MHz, DMSO- $\left.d_{6}\right) \delta: 13.04(\mathrm{~s}, 1 \mathrm{H}), 8.23(\mathrm{~s}, 2 \mathrm{H}), 7.47(\mathrm{~d}$, $J=164.0 \mathrm{~Hz}, 6 \mathrm{H}) ;{ }^{13} \mathrm{C}$ NMR $\left(100 \mathrm{MHz}\right.$, DMSO- $\left.d_{6}\right) \delta$ : $158.0,150.1,134.4,129.0,128.1,122.2,118.5,111.7$, 106.6, 106.5, 99.5; ESI-MS m/z: $229.1[\mathrm{M}+\mathrm{H}]^{+}$.

2-(4-溴苯基)- $1 H$-苯并 $[d]$ 咪唑(3e): 黄色固体, m.p. $295 \sim 296{ }^{\circ} \mathrm{C}$ (Lit. $\left.{ }^{[16]]}: 295 \sim 299{ }^{\circ} \mathrm{C}\right) ;{ }^{1} \mathrm{H}$ NMR $(500$ MHz, DMSO- $\left.d_{6}\right) \delta: 7.74(\mathrm{~d}, J=8.5 \mathrm{~Hz}, 2 \mathrm{H}), 7.67(\mathrm{~d}, J=$ $8.5 \mathrm{~Hz}, 1 \mathrm{H}), 7.49(\mathrm{~d}, J=8.4 \mathrm{~Hz}, 2 \mathrm{H}), 7.28 \sim 7.26(\mathrm{~m}, 1 \mathrm{H})$, $6.95(\mathrm{~d}, J=8.5 \mathrm{~Hz}, 1 \mathrm{H}), 5.58(\mathrm{~s}, 1 \mathrm{H}) ;{ }^{13} \mathrm{C}$ NMR $(126 \mathrm{MHz}$, DMSO- $\left.d_{6}\right) \delta: 152.6,143.1,132.3,132.2,131.5,129.7$, 128.8, 121.1, 119.9, 111.6, 47.4; ESI-MS m/z: $273.0[\mathrm{M}+$ $\mathrm{H}]^{+}$.

4-(1H-苯并 $[d]$ 咪唑-2-基)-2-甲氧基苯酚(3f)：白色 固体, m.p. $222 \sim 224{ }^{\circ} \mathrm{C}$ (Lit. ${ }^{[16 k]}: 221 \sim 222{ }^{\circ} \mathrm{C}$ ); ${ }^{1} \mathrm{H}$ NMR $\left(400 \mathrm{MHz}\right.$, acetone- $\left.d_{6}\right) \delta: 11.15(\mathrm{~s}, 1 \mathrm{H}), 8.10(\mathrm{~s}, 1 \mathrm{H})$, $7.84(\mathrm{~d}, J=4.0 \mathrm{~Hz}, 1 \mathrm{H}), 7.69(\mathrm{~d}, J=8.0 \mathrm{~Hz}, 1 \mathrm{H}), 7.55(\mathrm{dd}$, $J=6.0,3.2 \mathrm{~Hz}, 2 \mathrm{H}), 7.17(\mathrm{dd}, J=6.0,3.2 \mathrm{~Hz}, 2 \mathrm{H}), 6.96(\mathrm{~d}$, $J=8.0 \mathrm{~Hz}, 1 \mathrm{H}), 3.95$ (s, $3 \mathrm{H}) ;{ }^{13} \mathrm{C}$ NMR $(126 \mathrm{MHz}$, DMSO- $\left.d_{6}\right) \delta: 151.8,148.5,147.9,121.7,121.5,119.8$, $115.7,114.5,110.5,55.8,29.9,29.1,22.1,14.0$; ESI-MS $\mathrm{m} / \mathrm{z}: 241.1[\mathrm{M}+\mathrm{H}]^{+}$.

3-(1H-苯并 $[d]$ 咪唑-2-基)苯甲腈 (3g)：白色固体， m.p. $257 \sim 258{ }^{\circ} \mathrm{C}$ (Lit. ${ }^{[16 \mathrm{~d}]}: 257 \sim 259{ }^{\circ} \mathrm{C}$ ); ${ }^{1} \mathrm{H}$ NMR $(400$ MHz, acetonitrile- $\left.d_{3}\right) \delta: 10.96(\mathrm{~s}, 1 \mathrm{H}), 8.41(\mathrm{~s}, 1 \mathrm{H}), 8.35$ (d, $J=8.0 \mathrm{~Hz}, 1 \mathrm{H}), 7.82(\mathrm{~d}, J=8.0 \mathrm{~Hz}, 1 \mathrm{H}), 7.69$ (t, $J=8.0$ $\mathrm{Hz}, 1 \mathrm{H}$ ), 7.64 (dd, $J=6.0,3.2 \mathrm{~Hz}, 2 \mathrm{H}), 7.28$ (dd, $J=6.0$, $3.0 \mathrm{~Hz}, 2 \mathrm{H}) ;{ }^{13} \mathrm{C}$ NMR (100 MHz, DMSO- $\left.d_{6}\right) \delta: 149.15$, $143.31,134.85,132.97,131.31,130.82,130.23,129.68$, $122.55,118.34,112.12,59.69,20.68,14.01$; ESI-MS $m / z$ : $220.1[\mathrm{M}+\mathrm{H}]^{+}$.

2-(2-氟苯基)-1 $1 H$-苯并 $[d]$ 咪唑(3h)：白色固体，m.p. $200 \sim 203{ }^{\circ} \mathrm{C}$ (Lit. ${ }^{[16]}$ : $201 \sim 207{ }^{\circ} \mathrm{C}$ ); ${ }^{1} \mathrm{H}$ NMR $(400$ MHz, acetone- $\left.d_{6}\right) \delta$ : $11.68(\mathrm{~s}, 1 \mathrm{H}), 8.45(\mathrm{~d}, J=4.0 \mathrm{~Hz}$, $1 \mathrm{H}), 7.66(\mathrm{~d}, J=2.7 \mathrm{~Hz}, 2 \mathrm{H}), 7.58 \sim 7.52(\mathrm{~m}, 1 \mathrm{H}), 7.42 \sim$ $7.31(\mathrm{~m}, 2 \mathrm{H}), 7.24(\mathrm{dd}, J=4.0,4.0 \mathrm{~Hz}, 2 \mathrm{H}) ;{ }^{13} \mathrm{C}$ NMR $\left(100 \mathrm{MHz}\right.$, DMSO- $\left.d_{6}\right) \delta: 160.7,158.2,146.4,146.4,131.8$, $131.8,130.2,130.2,125.0,125.0,122.3,118.1,118.0$, 116.6, 116.4; ESI-MS m/z: $213.1[\mathrm{M}+\mathrm{H}]^{+}$.

2-(1H-苯并 $[d]$ 咪唑-2-基)苯酚(3i): 白色固体, m.p. $241 \sim 242{ }^{\circ} \mathrm{C}$ (Lit. $\left.{ }^{[16]}: 240 \sim 242{ }^{\circ} \mathrm{C}\right) ;{ }^{1} \mathrm{H}$ NMR $(400$ $\mathrm{MHz}$, acetonitrile- $\left.d_{3}\right) \delta: 13.16(\mathrm{~s}, 1 \mathrm{H}), 11.07(\mathrm{~s}, 1 \mathrm{H}), 7.80$ (d, $J=8.0 \mathrm{~Hz}, 1 \mathrm{H}), 7.64(\mathrm{~d}, J=28.0 \mathrm{~Hz}, 2 \mathrm{H}), 7.35$ (d, $J=$ $32.0 \mathrm{~Hz}, 3 \mathrm{H}), 7.06 \sim 7.00(\mathrm{~m}, 2 \mathrm{H}) ;{ }^{13} \mathrm{C}$ NMR $(100 \mathrm{MHz}$, DMSO- $\left.d_{6}\right) \delta: 158.0,151.7,140.8,133.1,131.7,126.2$, 123.2, 122.4, 119.1, 117.9, 117.1, 112.6, 111.5; ESI-MS $\mathrm{m} / \mathrm{z}: 221.1[\mathrm{M}+\mathrm{H}]^{+}$.

2 - 异丙基- $1 H$-苯并 $[d]$ 咪唑 (3jj): 黄色固体, m.p. $219 \sim 221{ }^{\circ} \mathrm{C}$ (Lit. ${ }^{[16 \mathrm{~m}]}: 220 \sim 225{ }^{\circ} \mathrm{C}$ ); ${ }^{1} \mathrm{H}$ NMR $(400$ $\mathrm{MHz}$, acetonitrile- $\left.d_{3}\right) \delta: 7.48(\mathrm{dd}, J=6.0,3.2 \mathrm{~Hz}, 2 \mathrm{H}), 7.15$ (dd, $J=6.0,3.2 \mathrm{~Hz}, 2 \mathrm{H}), 3.21 \sim 3.13(\mathrm{~m}, 1 \mathrm{H}), 1.38(\mathrm{~s}, 6 \mathrm{H})$; ${ }^{13} \mathrm{C}$ NMR (100 MHz, acetone- $d_{6}$ ) $\delta: 205.4,205.1,159.8$, 121.2, 114.3, 30.8, 20.9; ESI-MS $m / z: 161.1[\mathrm{M}+\mathrm{H}]^{+}$.

5,6 -二氯-2-苯基- $1 H$ - 苯并 $[d]$ 咪唑 (3k)：白色固 体, m.p. $220 \sim 221{ }^{\circ} \mathrm{C}$ (Lit. ${ }^{[161]}: 220 \sim 222{ }^{\circ} \mathrm{C}$ ); ${ }^{1} \mathrm{H}$ NMR (400 MHz, acetonitrile- $\left.d_{3}\right) \delta: 11.07(\mathrm{~s}, 1 \mathrm{H}), 8.07$ (d, $J=4.0$ $\mathrm{Hz}, 2 \mathrm{H}), 7.78$ (d, $J=56.0 \mathrm{~Hz}, 2 \mathrm{H}), 7.54(\mathrm{~s}, 3 \mathrm{H}) ;{ }^{13} \mathrm{C} \mathrm{NMR}$ $\left(100 \mathrm{MHz}, \mathrm{DMSO}-d_{6}\right) \delta: 153.79,143.45,134.52,130.48$, 129.26, 129.00, 126.70, 124.69, 124.19, 119.91, 112.64; ESI-MS $m / z: 264.1[\mathrm{M}+\mathrm{H}]^{+}$.

5-硝基-2-苯基- $1 H$-苯并 $[d]$ 咪唑(3I)：黄色固体, m.p. 
$205 \sim 208{ }^{\circ} \mathrm{C}$ (Lit. $\left.{ }^{[16 n]}: 208 \sim 210{ }^{\circ} \mathrm{C}\right) ;{ }^{1} \mathrm{H}$ NMR $(400$ $\left.\mathrm{MHz}, \mathrm{DMSO}-d_{6}\right) \delta$ : $13.61(\mathrm{~s}, 1 \mathrm{H}), 8.50(\mathrm{~s}, 1 \mathrm{H}), 8.18(\mathrm{dd}$, $J=40.0,8.0 \mathrm{~Hz}, 3 \mathrm{H}), 7.60(\mathrm{~d}, J=8.0 \mathrm{~Hz}, 4 \mathrm{H}) ;{ }^{13} \mathrm{C} \mathrm{NMR}$ $\left(100 \mathrm{MHz}, \mathrm{DMSO}-d_{6}\right) \delta: 155.6,142.6,132.7,130.8,129.3$, 129.0, 128.5, 127.0, 117.8, 114.6, 111.6; ESI-MS $\mathrm{m} / \mathrm{z}$ : $240.1[\mathrm{M}+\mathrm{H}]^{+}$.

5-甲基-2-苯基- $1 H$-苯并 $[d]$ 咪唑 (3m)：白色固体, m.p. $240 \sim 241{ }^{\circ} \mathrm{C}$ (Lit. ${ }^{[16 f]}: 246{ }^{\circ} \mathrm{C}$ ); ${ }^{1} \mathrm{H}$ NMR (400 MHz, acetonitrile- $\left.d_{3}\right) \delta: 11.99(\mathrm{~s}, 1 \mathrm{H}), 8.07(\mathrm{~d}, J=8.0 \mathrm{~Hz}, 2 \mathrm{H})$, $7.51(\mathrm{dt}, J=13.4,7.2 \mathrm{~Hz}, 4 \mathrm{H}), 7.40$ (s, 1H), 7.07 (d, $J=$ $8.0 \mathrm{~Hz}, 1 \mathrm{H}), 2.46(\mathrm{~s}, 3 \mathrm{H}) ;{ }^{13} \mathrm{C}$ NMR (100 MHz, DMSO- $\left.d_{6}\right)$ $\delta: 150.78,135.33,131.90,130.37,129.67,128.94,126.35$, 124.02, 123.29, 118.50, 111.08, 21.43; ESI-MS m/z: 209.1 $[\mathrm{M}+\mathrm{H}]^{+}$.

2-(吡啶-2-基)-1H-苯并 $[d]$ 咪唑(3n): 白色固体, m.p. $218 \sim 219{ }^{\circ} \mathrm{C}$ (Lit. ${ }^{[16 n]}$ : $\left.216 \sim 219{ }^{\circ} \mathrm{C}\right) ;{ }^{1} \mathrm{H}$ NMR (400 $\mathrm{MHz}$, acetone- $\left.d_{6}\right) \delta: 12.06(\mathrm{~s}, 1 \mathrm{H}), 8.70 \sim 8.64(\mathrm{~m}, 1 \mathrm{H})$, $8.41(\mathrm{~d}, J=8.0 \mathrm{~Hz}, 1 \mathrm{H}), 7.97$ (td, $J=7.8,1.7 \mathrm{~Hz}, 1 \mathrm{H}), 7.68$ (dd, $J=5.6,3.4 \mathrm{~Hz}, 2 \mathrm{H}), 7.46$ (ddd, $J=7.6,4.8,1.0 \mathrm{~Hz}$, $1 \mathrm{H}), 7.25$ (dd, $J=6.0,3.2 \mathrm{~Hz}, 2 \mathrm{H}) ;{ }^{13} \mathrm{C} \mathrm{NMR}(100 \mathrm{MHz}$, DMSO- $\left.d_{6}\right) \delta: 150.8,149.4,148.5,139.4,137.6,124.8$, 122.6, 121.5, 115.7; ESI-MS m/z: $196.1[\mathrm{M}+\mathrm{H}]^{+}$.

2-(4-硝基苯基)- $1 H$-苯并 $[d]$ 咪唑(3o): 黄色固体, m.p. 310 311 ${ }^{\circ} \mathrm{C}$ (Lit. ${ }^{[160]}$ : 311 $\left.312{ }^{\circ} \mathrm{C}\right)$; ${ }^{1} \mathrm{H}$ NMR (400 $\left.\mathrm{MHz}, \mathrm{DMSO}-d_{6}\right) \delta: 13.22(\mathrm{~s}, 1 \mathrm{H}), 8.34(\mathrm{~s}, 4 \mathrm{H}), 7.59$ (d, $J=40.0 \mathrm{~Hz}, 2 \mathrm{H}), 7.20(\mathrm{~s}, 2 \mathrm{H}) ;{ }^{13} \mathrm{C} \mathrm{NMR}(100 \mathrm{MHz}$, DMSO- $\left.d_{6}\right) \delta: 149.0,147.8,143.8,136.0,135.3,130.3$, 127.4, 124.2, 122.4, 119.5, 111.8; ESI-MS m/z: 229.1 $[\mathrm{M}+\mathrm{H}]^{+}$.

2-(噻吩-2-基)- $1 H$-苯并 $[d]$ 味唑(3p): 黄色固体, m.p. $341 \sim 342{ }^{\circ} \mathrm{C}$ (Lit. ${ }^{[16 p]}$ : $\left.342 \sim 343{ }^{\circ} \mathrm{C}\right) ;{ }^{1} \mathrm{H}$ NMR $(500$ $\left.\mathrm{MHz}, \mathrm{CDCl}_{3}\right) \delta: 11.15(\mathrm{~d}, J=3.09 \mathrm{~Hz}, 1 \mathrm{H}), 8.33 \sim 8.27$ $(\mathrm{m}, 2 \mathrm{H}), 7.42 \sim 7.34(\mathrm{~m}, 2 \mathrm{H}), 7.81(\mathrm{dd}, J=6.01,3.03 \mathrm{~Hz}$, $1 \mathrm{H}), 7.56(\mathrm{~d}, J=5.43 \mathrm{~Hz}, 2 \mathrm{H}) ;{ }^{13} \mathrm{C} \mathrm{NMR}(100 \mathrm{MHz}$, $\left.\mathrm{CDCl}_{3}\right) \delta: 131.5,128.9,127.6,127.2,125.1,124.6,120.0$, 110.6; ESI-MS m/z: $200.1[\mathrm{M}+\mathrm{H}]^{+}$.

辅助材料(Supporting Information) 化合物 $\mathbf{3 a} \sim \mathbf{3 p}$ 的 ${ }^{1} \mathrm{H}$ NMR 和 ${ }^{13} \mathrm{C}$ NMR 谱图. 这些材料可以免费从本刊网 站(http://sioc- journal.cn/)上下载.

\section{References}

[1] (a) Zhu, G.; Yang, L.; Cui, D. Chin. J. Org. Chem. 2014, 34, 495 (in Chinese).

(朱观明, 杨柳阳, 崔冬梅, 有机化学, 2014, 34, 495.)

(b) Dhanamjayulu, P.; Boga, R. B.; Mehta, A. Toxicon 2019, 170, 60 . (c) Ghadari, R.; Namazi, H.; Aghazadeh, M. Appl. Organomet. Chem. 2018, 32, 3965.

(d) Horton, D. A.; Bourne, G. T.; Smythe, M. L. Chem. Rev. 2003, 103, 893.

(e) Ziarati, A.; Badiei, A.; Ziarani, G. M.; Eskandarloo, H. Catal. Commun. 2017, 95, 77

(f) Ashok, D.; Reddy, M. R.; Nagaraju, N.; Dharavath, R.; Ramakrishna, K.; Gundu, S.; Shravani, P.; Sarasija, M. Med. Chem. Res. 2020, 29, 699

[2] (a) Algul, O.; Kaessler, A.; Apcin, Y.; Yilmaz, A.; Jose, J. Molecules 2008, 13, 736

(b) Khattab, M.; Galal, S. A.; Ragab, F. A. F.; Diwani, H. I. E. Res. Chem. Intermed. 2013, 39, 2917.

(c) Khalili, S. B.; Sardarian, A. R. Monatsh. Chem. 2012, 143, 841. (d) Hu, Z.; Zhao, T.; Wang, M.; Wu, J.; Yu, W.; Chang, J. J. Org. Chem. 2017, 82, 3152 .

(e) Wang, S.; Guan, L.; Zang, J.; Xing, K.; Zhang, J.; Liu, D.; Zhao, L. Molecules 2019, 24, 1198.

[3] (a) Khosravi, K.; Kazemi, S. Chin. Chem. Lett. 2012, 23, 61.

(b) Zhang, C.; Zhang, L.-R.; Jiao, N. Green Chem. 2012, 14, 3273.

(c) Shelkar, R.; Sarode, S.; Nagarkar, J. Tetrahedron Lett. 2013, 54, 6986.

[4] (a) Rostamizadeh, S.; Amani, A. M.; Aryan, R.; Ghaieni, H. R.; Norouzi, L. Monatsh. Chem. 2009, 140, 547.

(b) Brasche, G.; Buchwald, S. L. Angew. Chem., Int. Ed. 2008, 120 , 1958.

(c) Martins, G. M.; Puccinelli, T.; Gariani, R. A.; Xavier, F. R.; Silveira, C. C.; Mendes, S. R. Tetrahedron Lett. 2017, 58, 1969.

(d) Nale, D. B.; Bhanage, B. M. Synlett 2015, 26, 2835.

(e) De Luca, L.; Porcheddu, A. Eur. J. Org. Chem. 2011, 5791.

[5] (a) Diao, X.; Wang, Y.; Jiang, Y.; Ma, D. J. Org. Chem. 2009, 74, 7974.

(b) Xiao, Q.; Wang, W.-H.; Liu, G.; Meng, F.-K.; Chen, J.-H.; Yang, Z.; Shi, Z.-J. Chem.-Eur. J. 2009, 15, 7292.

(c) Deng, X.; Mani, N. S. Eur. J. Org. Chem. 2010, 2010, 680

(d) Wray, B. C.; Stambuli, J. P. Org. Lett. 2010, 12, 4576.

(e) Bhagat, S. B.; Ghodse, S. M.; Telvekar, V. N. J. Chem. Sci. 2018, 130, 10.

[6] Qu, Y.; Pan, L.; Wu, Z.; Zhou, X. Tetrahedron 2013, 69, 1717.

[7] Xiang, S.-K.; Zhang, D.-X.; Hu, H.; Shi, J.-L.; Guo, L.-G.; Feng, C.; Wang, B.-Q.; Zhao, K.-Q.; Hu, P.; Yang, H.; Yu, W.-H. Adv. Synth. Catal. 2013, 355, 1495.

[8] (a) Wasilke, J.-C.; Obrey, S. J.; Baker, R. T.; Bazan, G. C. Chem. Rev. 2005, 105, 1001 .

(b) Wu, L.; Wang, L.; Chen, P.; Guo, Y.-L.; Liu, G. Adv. Synth. Catal. 2020, 362, 2189.

(c) Niknam, E.; Panahi, F.; Khalafi-Nezhad, A. Eur. J. Org. Chem. 2020, 2020, 2699.

[9] (a)Yu, J.; Xia, Y.; Lu, M. Appl. Organomet. Chem. 2014, 28, 764. (b) Cao, Z.; Zhu, Q.; Lin, Y.-W.; He, W.-M. Chin. Chem. Lett. 2019, 30, 2132

[10] Fujishima, A.; Honda, K. Nature (London) 1972, 238, 37.

[11] (a) Zeng, F.-L.; Sun, K.; Chen, X.-L.; Yuan, X.-Y.; He, S.-Q.; Liu, Y.; Peng, Y.-Y.; Qu, L.-B.; Lv, Q.-Y.; Yu, B. Adv. Synth. Catal. 2019, 361, 5176

(b) Samanta, S.; Khilari, S.; Srivastava, R. ACS Appl. Nano Mater. 2018, 1,426

(c) Li, Z.; Song, H.; Guo, R.; Zuo, M.; Hou, C.; Sun, S.; He, X.; Sun, Z.; Chu, W. Green Chem. 2019, 21, 3602.

(d) Chen, D.; Liu, J.; Zhang, X.; Jiang, H.; Li, J. Chin. J. Org. Chem. 2019, 39, 3353 (in Chinese).

(陈丹, 刘剑沉，张馨元，蒋合众，李加洪，有机化学，2019，39, 3353.)

(e) Peng, S.; Song, Y.-X.; He, J.-Y.; Tang, S.-S.; Tan, J.-X..; Cao, Z.; Lin, Y.-W.; He W.-M. Chin. Chem. Lett. 2019, 30, 2287.

(f) Xie, L.-Y.; Fang, T.-G.; Tan, J.-X.; Zhang, B.; Cao, Z.; Yang, L.-H.; He, W.-M. Green Chem. 2019, 21, 3858.

(g) Xie, L.-Y.; Liu, Y.-S.; Ding, H.-R.; Gong, S.-F.; Tan, J.-X.; He, 
J.-Y.; Cao, Z.; He, W.-M. Chin. J. Catal. 2020, 41, 1168

[12] (a) Li, L.; Huang, M.; Liu, C.; Xiao, J. C.; Chen, Q. Y.; Guo, Y.; Zhao, Z. G. Org. Lett. 2015, 17, 4714.

(b) Zhang, M.; Ruzi, R.; Li, N.; Xie, J.; Zhu, C. Org. Chem. Front. 2018, 5,749 .

(c) Hou, H.; Zhu, S.; Pan, F.; Rueping, M. Org. Lett. 2014, 16, 2872.

[13] Jana, S.; Verma, A.; Kadu, R.; Kumar, S. Chem. Sci. 2017, 8, 6633.

[14] (a) Ke, F.; Xu, Y.; Zhu, S.; Lin, X.; Lin, C.; Zhou, S.; Su, H. Green Chem. 2019, 21, 4329.

(b) Ke, F.; Liu, C.; Zhang, P.; Xu, J.; Chen, X. Synth. Commun. 2018, 48, 3089.

[15] (a) Tian, W.; Xu, S.; Liang, Z.; Sun, D.; Zhang, R. Chin. J. Org. Chem. 2016, 36, 2121 (in Chinese).

(田文艳, 徐松, 梁中卫, 孙德立, 张荣华, 有机化学, 2014, 36, 2121.)

(b) Jiang, W.-J.; Song, Y.-T.; Wei, X.-J.; Xu, Y.; Lu, J.; Jiang, B.; Hao, W.-J. Chin. J. Org. Chem. 2019, 39, 1095 (in Chinese).

(蒋文婕, 宋雅婷, 韦晓静, 徐义, 陆娟, 姜波, 郝文娟, 有机化 学, 2019, 39, 1095.)

(c) Yue, H.-L.; Bao, P.-L.; Wang, L.-L.; Lü, X.-X.; Yang, D.-S.; Wang, Y.; Wei, W. Chin. J. Org. Chem. 2019, 39, 463 (in Chinese). (岳会兰, 鲍鹏丽, 王雷雷, 吕晓霞, 杨道山, 王桦, 魏伟, 有机 化学, 2019, 39, 463.)

(d) Peng, S.; Hu, D.; Hu, J.-L.; Lin, Y.-W.; Tang, S.-S.; Tang, H.-S. He, J.-Y.; Cao, Z.; He, W.-M. Adv. Synth. Catal. 2019, 361, 5721.

[16] (a) Chen, C.; Chen, C.; Li, B.; Tao, J.; Peng, J. Molecules 2012, 17, 12506.

(b) Rahim, G.; Namazi, H.; Aghazadeh, M. Appl. Organomet. Chem. 2018, 32, e3965.

(c) Ghosh, P.; Subba, R. Tetrahedron Lett. 2015, 56, 2691. (d) Qiu, D.; Wei, H.; Zhou, L.; Zeng, Q. Appl. Organomet. Chem. 2014, 28, 109.

(e) Alapati, M. L. P. R.; Abburi, S. R.; Mukkamala, S. B.; Rao Krishnaji, M. Synth. Commun. 2015, 45, 2436.

(f) Sharghi, H.; Asemani, O.; Khalifeh, R. Synth. Commun. 2008 , 38,1128 .

(g) Sun, P.; Hu, Z. J. Heterocycl. Chem. 2006, 43, 773.

(h) Park, S.; Jung, J.; Cho, E. J. Eur. J. Org. Chem. 2014, 4148.

(i) Ravi, O.; Shaikh, A.; Upare, A.; Singarapu, K. K.; Bathula, S. R. J. Org. Chem. 2017, 82, 4422.

(j) Itoh, T.; Nagata, K.; Ishikawa, H.; Ohsawa, A. Heterocycles 2004, 63, 2769.

(k) Panda, S. S.; Jain, S. Synth. Commun. 2011, 41, 729.

(l) Sandera, G.; Isensee, R. W.; Joseph, L. J. Am. Chem. Soc. 1954, 76, 5173 .

(m) Wallace, J. M.; Soederberg, B. C. G.; Tamariz, J.; Akhmedov, N. G.; Hurley, M. T. Tetrahedron 2008, 41, 9675.

(n) Murugulla, A. C.; Donthabhakthuni, S.; Takehiko, S. Tetrahedron Lett. 2011, 52, 5575.

(o) Mao, Z.; Wang, C.; Song, X.; Mo, Y. Chin. J. Org. Chem. 2009, 29, 985 (in Chinese)

(毛郑州, 汪朝阳, 宋秀美, 莫阳青, 有机化学, 2009, 29, 985.)

(p) Kim, Y.; Kumar, M. R.; Park, N.; Heo, Y.; Lee, S. J. Org. Chem. 2011, 76, 9577.

[17] (a) Sharghi, H.; Hosseini Sarvari, M.; Moeini, F. Can. J. Chem. 2008, 86, 1044.

(b) Sharghi, H.; Aberi, M.; Doroodmand, M. M. Adv. Synth. Catal. 2008, 350, 2380

(c) Chen, Y. X.; Qian, L. F.; Zhang, W.; Han, B. Angew. Chem., Int. Ed. 2008, 120, 9470.

(Li, L.; Fan, Y.) 